$\begin{array}{cl}\begin{array}{c}\text { Revue } \\ \text { del'histoire } \\ \text { des religions }\end{array} & \text { Revue de l'histoire des religions } \\ & \begin{array}{l}\mid 2008 \\ \text { Varia }\end{array}\end{array}$

\title{
Aux sources du monachisme oriental
}

Abraham de Kashkar et le développement de la légende de Mar Awgin

On the track of Eastern Monasticism. Abraham of Kashkar and the development of the legend of Mar Awgin

\section{Florence Jullien}

\section{OpenEdition Journals}

\section{Édition électronique}

URL : http://journals.openedition.org/rhr/5913

DOI : $10.4000 /$ rhr.5913

ISSN : 2105-2573

\section{Éditeur}

Armand Colin

\section{Édition imprimée}

Date de publication : 1 mars 2008

Pagination : 37-52

ISBN : 978-2200-92443-0

ISSN : 0035-1423

\section{Référence électronique}

Florence Jullien, «Aux sources du monachisme oriental », Revue de l'histoire des religions [En ligne], 1 | 2008, mis en ligne le 01 mars 2011, consulté le 03 mai 2019. URL : http://journals.openedition.org/ rhr/5913 ; DOl : 10.4000/rhr.5913 


\section{Aux sources du monachisme oriental Abraham de Kashkar et le développement de la légende de Mar Awgin}

À examiner de près la documentation syriaque relative à Mar Awgin, figure emblématique du monachisme oriental (considéré comme l'initiateur de la vie monastique en Syrie et en Mésopotamie au Ive siècle), nous constatons des interpénétrations de traditions avec l'histoire d'Abraham de Kashkar: certains éléments de sa vie et de son action semblent avoir servi de support à la construction et à l'enrichissement de la légende eugénienne. La figure d'Awgin, progressivement élaborée alors que se développe l'historiographie sur le personnage d'Abraham et son æuvre de réforme qui inaugurait un véritable renouveau pour tout le monachisme en Perse, apparaît en partie comme un décalque du «Père des moines de l'Orient » Abraham, qu'il finit par éclipser.

\section{On the track of Eastern Monasticism. Abraham of Kashkar and the development of the legend of Mar Awgin}

From syriac documentation concerning Mar Awgin, emblematic figure of eastern monasticism (considered as the initiator of the monastic life in Syria and Mesopotamia in the IVth century), we notice confusions of traditions with the history of Abraham Kashkaraya: some biographical elements of his life and action may have been used as a medium for enhancing and constructing the tradition of Mar Awgin. Progressively elaborated while grew the historiography about Abraham and his work of reform - which caused a real revival for monasticism in Persia - Awgin partly appears as an image of the "Father of the monks of the Orient" Abraham, he finally eclipsed. 
Mar Awgin ${ }^{1}$, figure emblématique du monachisme oriental, considéré comme l'initiateur de la vie monastique en Syrie et en Mésopotamie au IV siècle, est devenu un référent hagiographique : les auteurs d'histoires monastiques, les biographes de moines célèbres, se sont plu à le faire côtoyer leurs protagonistes. Ainsi en est-il d'Abraham de Kashkar considéré comme «le Père des moines de l'Orient » par les communautés chrétiennes de l'Église de Perse. À examiner de près la documentation syriaque, nous constatons des interpénétrations de traditions qui nous conduisent à nous interroger sur l'influence de la vie et de l'œuvre d'Abraham sur le développement de la légende eugénienne.

Avant de devenir le fondateur du Grand monastère au mont Izla au milieu du $\mathrm{VI}^{\mathrm{e}}$ siècle et l'artisan de la réforme du monachisme en Iran sassanide ${ }^{2}$, Abraham, originaire de Kashkar, au cœur du BethAramaye, suivit un parcours de formation et d'initiation à la vie monastique qui le conduisit naturellement à l'école de sa ville : le Livre de la chasteté d'Isho'denah précise que dans son enfance, il fréquenta d'abord les écoles de Kashkar ${ }^{3}$. À cette époque, Kashkar était alors un centre d'enseignement assez fameux, et cette renommée devait se maintenir jusqu'à la fin de l'époque sassanide : Rabban Narsaï, qui y enseignait dans le premier quart du VII ${ }^{e}$ siècle, était réputé l'un des plus grands docteurs de son temps ${ }^{4}$. Par la suite, Abraham se rendit à l'école de Nisibe ; d'après la Chronique de Séert, le jeune étudiant se forma auprès d'Abraham l'Interprète et de Yohannan ${ }^{5}$, entre 544 et 547 (date du décès de ce dernier). Sa

1. Par commodité, j'ai choisi une transcription simple, en évitant les signes diacritiques.

2. Voir F. Jullien, Le monachisme en Perse. La réforme d'Abraham le Grand, 2008, sous presse.

3. J.-B. Chabot, Chasteté, p. 4; trad. p. 8, § 14.

4. J.M. Fiey, Dictionnaire, col. 1021. Nous lisons dans la Chronique de Séert que le futur métropolite de Nisibe Grégoire, originaire de Kashkar, y implanta deux écoles (deuxième moitié du $\mathrm{VI}^{\mathrm{e}}$ siècle) : prières et jeûnes alternaient avec des missions d'évangélisation dans le but de former les habitants mais aussi de lutter contre le paganisme, A. Scher, Chronique de Séert II/2, p. 508 [188].

5. A. Scher, Chronique de Séert II/1, p. 133 [41] ; J.-B. Chabot, Chasteté, p. 7, trad. p. 8, § 14. La Chronique de Séert les mentionne tous deux comme disciples de Narsaï, op. cit., p. 115 [23]-116 [24]. 
fondation allait devenir le principal pôle monastique pour la chrétienté syro-orientale, modèle qui connaîtra une diffusion sans précédent.

On relève plusieurs points biographiques communs entre Abraham et Awgin : missions d'évangélisation auprès des populations païennes ${ }^{6}$, parcours d'initiation anachorétique - relevant peut-être du topos littéraire - en Égypte et au Sinaï ${ }^{7}$. Tous deux font aussi une expérience en solitude, et furent directement associés, nous allons le voir, à la figure prestigieuse de Jacques de Nisibe.

\section{LES SUPERPOSITIONS DE TRADITIONS}

Au retour de ce périple d'apprentissage, Abraham choisit de s'installer au mont Izla dans le Tur 'Abdin et habita dans une grotte. Isho'denah ne parle pas de ce temps d'érémitisme; en revanche, l'historien monastique Thomas de Marga au IX siècle évoque une installation dans ce que le texte syriaque appelle une " grotte cachette » (mtshyt') à Izla, non loin de la ville de Nisibe ${ }^{8}$. La Chronique de Séert identifie cette cavité à "la grotte qu'avait jadis occupée Ja'qub, métropolite de Nisibe $»^{9}$. Ja'qub avait en effet mené une vie anachorétique sur les hauteurs de Singar avant d'être choisi pour occuper le siège épiscopal de Nisibe vers 308. D'après la Vita d'Abraham (éditée par F. Nau en 1918-1919 à partir du manuscrit de Berlin syr. 175, et attribuée à Babaï le Grand, troisième abbé du Grand monastère), cette caverne se situait en un lieu-dit $m d r$ ', Madra ${ }^{10}$. La Vie syriaque de Ja'qub de Nisibe, publiée en 1894 par P. Bedjan dans la collection des Acta martyrum et sanctorum, édition

6. Isho 'denah relève qu'Abraham «prêcha beaucoup parmi les païens de [Hira] », J.-B. Chabot, Chasteté, p. 7; trad. p. 8, § 14.

7. J.-B. Chabot, Chasteté, p. 7 ; trad. p. 8, § 14. E. Budge, The Book of Governors, I, p. 23 ; II, p. 39. F. Jullien, Le monachisme en Perse, 2008, chapitre 2.

8. E. Budge, The Book of Governors, I, p. 23; II, p. 40.

9. A. Scher, Chronique de Séert II/1, p. 134 [42].

10. F. Nau, «Histoire d'Abraham de Kaskar », p. 170. Le nom de Marda apparaît dans la règle d'Abraham, comme toponyme du site du Grand monastère, sous la graphie $m r d$ ', sans doute une métathèse pour $m d r$ '. A. Vööbus, Syriac and Arabic Documents, 1960, p. 165. 
basée sur le manuscrit de la British Library Add. $12174^{11}$, ne confirme pas ce toponyme. Dans un passage, il est question de la grotte dans laquelle se retirait Ja'qub au plus fort de l'hiver, pour $\mathrm{s}^{\prime} \mathrm{y}$ abriter quelques jours ${ }^{12}$; cette caverne se trouvait sur les pentes des hautes montagnes des environs de Nisibe. L'auteur du texte semble suggérer que Ja 'qub avait coutume de parcourir la région au creux des vallées encaissées; seule la rigueur des intempéries hivernales le contraignait à se réfugier dans cet abri - toujours le même, semble-t-il. Le texte syriaque restitue en fait les données de l'Histoire philothée de Théodoret de Cyr, dont il constitue une traduction abrégée. L'évêque de Cyr parle de cette caverne habitée durant la saison froide, Ja'qub séjournant dans les taillis avec «le ciel pour toit » durant le reste de l'année ${ }^{13}$.

L'association, anachronique, de ces deux figures relève naturellement d'un développement hagiographique. Le médiat de Jacques de Nisibe laisse en tout cas entrevoir comme une chaine cohérente rattachant Abraham de Kashkar aux traditions des origines mêmes du monachisme en ces contrées ; ainsi se trouve-t-il, par le truchement de cet élément comparatif, intégré dans une tradition locale préexistant à la fondation d'Izla, située précisément dans la région de Nisibe. Originaire de Kashkar, Abraham s'installe à Izla sous juridiction nisibéenne : la relation établie par les sources avec le métropolite de Nisibe a-t-elle pour objectif de renforcer l'ancrage d'Abraham dans cette région? On se rappelle qu' Isaac de Ninive, du Beth-Qatraye, ne fut pas accepté comme évêque par ses diocésains en raison de ses origines; le Livre de la chasteté évoque les jalousies des " gens de l'intérieur » à l'encontre du nouveau venu ${ }^{14}$. Ces interférences avec la tradition jacobienne par feuilletages successifs tendaient à conférer à Abraham, avant même son œuvre fondatrice, une ascendance susceptible d'authentifier sa position à

11. P. Bedjan, Acta Martyrum IV, p. 262-273. W. Wright, Catalogue British Museum, $\mathrm{n}^{\circ}$ DCCCCLX, 45, p. 1131.

12. P. Bedjan, Acta Martyrum IV, p. 263-264. P. Peeters, "Jacques de Nisibe », p. 285-373.

13. P. Canivet, A. Leroy-Molinghen, Théodoret de Cyr, p. 162-163. Traduction de la Vie syriaque en préparation par M. Debié et alii.

14. J.-B. Chabot, Chasteté, p. 63-64 ; trad. p. 53-54, § 124. 
venir de paternité spirituelle sur tout le monachisme de l'Orient mésopotamien et perse.

Les liens qu'établit la Chronique de Séert entre Ja 'qub de Nisibe et Abraham trouvent un parallèle en ceux, plus tardifs cependant, de Ja'qub et de Mar Awgin, repérables chez certains auteurs syriaques : ainsi, dans sa liste des métropolitains de Nisibe, Élie Bar Shinaya (XI ${ }^{\mathrm{e}}$ siècle) présente-t-il Ja'qub comme un ermite qui s'initia à la vie ascétique à l'école d'Awgin ${ }^{15}$. Le texte syriaque de la Vie d'Awgin, postérieur au $\mathrm{X}^{\mathrm{e}}$ siècle, insiste dans sa deuxième partie sur les rapports amicaux qui unissaient ce moine à Ja ${ }^{6}$ qub $^{16}$ : P. Peeters a montré comment l'auteur de cette Vie aurait introduit à dessein la figure populaire de Ja'qub de Nisibe, qui bénéficiait alors d'une très grande renommée, pour glorifier son protagoniste donné comme contemporain du grand saint ${ }^{17}$. Or, la sainteté de l'un rejaillit progressivement sur l'autre, et Ja 'qub devient ainsi un fidèle d' Awgin maitre avisé, dispensateur de sagesse, conseiller inspiré et prophétique. Le texte rattache même à Awgin l'évangélisation de Nisibe - dont le siège épiscopal, on le sait, fut fondé par Ja'qub.

Cette association d'Awgin avec Ja 'qub trouve un écho dans une superposition biographique entre Abraham et Awgin qui vécut dans la solitude au lieu même que choisit Abraham pour s'établir, le mont Izla. L'occupation de la caverne de Ja'qub par Abraham aurait-elle inspiré par la suite un développement de la légende eugénienne, rattachant cette fois-ci Awgin à cette grotte ${ }^{18}$ ?

15. E.W. Brooks, Eliae metropolitae Nisibeni, p. 98 ; trad. p. 47. P. Peeters, « Jacques de Nisibe », p. 290-291.

16. Ja'qub (dont l'histoire tait l'identification avec l'évêque de Nisibe), parti à la recherche du bois de l'arche de Noé, prit conseil auprès de l'ascète qui l'envoya en Cordouène. Dans les montagnes, il retrouva un morceau de l'arche et l'apporta à Awgin qui en fit une croix; Ja'qub invita ensuite l'ermite à l'inauguration de son monastère, édifié sur l'emplacement même de la relique enfouie, P. Bedjan, Acta Martyrum III, p. 435-437. J.M. Fiey, Nisibe, p. 221.

17. P. Peeters, « Jacques de Nisibe », p. 339.

18. Notons que les diptyques nestoriens du XIV e siècle ont conservé, dans la sixième section dévolue aux moines et aux solitaires, une rubrique pour les moines locaux de la région de Nisibe, J.M. Fiey, «Diptyques», p. 395: « Sur les fondements des Douze et des Soixante-dix ont bâti Mar Awgin, Mar Shallita, Mar Ja'qub le Juste de Nisibe, Mar Abraham, Mar Ahha et Mar Yohannan ». « Ja'qub le Juste » renvoie à une appellation des temps apostoliques, désignation du frère du Seigneur, évêque de Jérusalem. 


\section{Abraham et Le dÉvelopPement de La LÉGENDE EUGÉNIENNE}

La question de la paternité eugénienne pour le monachisme oriental avait été fort bien approfondie par J. Labourt dans son chapitre consacré à Awgin, puis par J. M. Fiey qui, dans ses études de 1962 et 1970, a montré de façon irréfutable les origines relativement tardives de la légende. Sur base de ces acquis, nous voudrions examiner le bien-fondé des liens que les sources établissent entre cette tradition hagiographique eugénienne et certains éléments de l'histoire d'Abraham qui semblent avoir servi de support - et c'est là notre hypothèse - à la construction et à l'enrichissement d'une tradition des origines monastiques autour d'Awgin.

\section{La tradition littéraire}

Il nous faut rappeler en préalable les données chronologiques relatives au personnage. J. Labourt fut le premier à constater qu' aucune mention d'Awgin ne se trouvait dans la littérature avant le $\mathrm{IX}^{\mathrm{e}}$ siècle ${ }^{19}$ : c'est entre Thomas de Marga, qui n'en parle pas, et Isho'denah de Basra, qui lui consacre la première notice de son Livre de la chasteté, qu'il estime devoir placer la formation de l'histoire d'Awgin, ou du moins sa vulgarisation ${ }^{20}$. Pour J. M. Fiey, le Livre de la chasteté montrerait un état de la légende d'Awgin en évolution : le personnage n'est pas encore considéré comme le Père du monachisme et le nombre de ses disciples est restreint. Il conjecture qu' Isho 'denah aurait pu avoir connaissance de ce cycle eugénien par l'intermédiaire du couvent placé sous le vocable de Yonan d'Anbar, nom de l'un des principaux disciples d'Awgin, que le métropolite de Basra paraît avoir tout particulièrement estimé puisqu'il lui consacra un poème ${ }^{21}$. Ajoutons que l'Histoire en vers du couvent de Sabrisho“

19. J. Labourt, Le christianisme, p. 310-311. Cf. S. Brock, "Early Syrian monasticism", p. 3.

20. J. Labourt, op. cit., p. 308.

21. J.M. Fiey, «Aonès », p. 71 et n. 5 . La version du Livre de la chasteté détruite lors de l'autodafé de Diamper en Inde en 1599 commençait directement avec Abraham de Kashkar, négligeant la figure d'Awgin. J.M. Fiey, « Ichô‘dnah », p. 446-447. J.-B. Chabot, «L'autodafé », p. 619-623. 
de Beth-Qoqa, datée de 820 par A. Mingana, comporte une mention d'Awgin dans son paragraphe introductif ${ }^{22}$. Les sources concernant la vie d'Awgin sont à présent bien connues : le Livre de la chasteté, les annalistes arabes chrétiens médiévaux Mari, 'Amr et Sliba, Barhebraeus, et les manuscrits présentant sa biographie (publiée en 1892 dans les Acta martyrum et sanctorum syriace par P. Bedjan ${ }^{23}$ ).

\section{Le couvent de Mar Awgin}

Si la tradition littéraire concernant Mar Awgin est assez tardive, des éléments locaux rattachés au mont Izla - base probable des développements ultérieurs de la légende - sont en revanche repérables plus tôt. Attardons-nous un instant sur le nom d'Awgin attribué à un couvent d'Izla : qu'en est-il exactement de l'ancienneté du vocable de ce monastère ? Grâce aux recherches d'I. Armalet restituées par J. M. Fiey dans son ouvrage sur la métropole de Nisibe, nous savons qu'un moine du nom de Mar Sallara, dont la biographie est restée inédite, avait été abbé au monastère de Mar Awgin de 643 jusqu'à sa mort, probablement vers $664^{24}$. I. Armalet présenta dans la revue al-Mashriq un résumé de la Vie syriaque de Mar Sallara, qu'il avait pu consulter lors d'un voyage dans le Tur 'Abdin en $1912^{25}$. S. Brock a retrouvé ce texte au monastère de Mar Gabriel de Qartamin, dans un manuscrit datant de $1963^{26}$; l'auteur anonyme attribue à cet abbé l'édification de vingt cellules. Il est difficile de tirer des conclusions fiables de ces manuscrits, non daté pour l'un, et tardif pour l'autre ${ }^{27}$.

22. A. Mingana, « Couvent de Sabrisho“», p. 173 ; p. 223.

23. P. Bedjan, Acta Martyrum III, p. 376-480.

24. Sallara fut formé, d'après ce texte, auprès de Yohannan le bédouin - que la notice actuelle du Livre de la chasteté présente comme un disciple d'Awgin, bien que ce rattachement reste sujet à caution. En effet, J.M. Fiey souligne que dans la notice consacrée à Yohannan par Isho'denah dans le même Livre, Awgin n'est pas cité ; pareillement, le fait que sa notice suive celle des deux sœurs de Mar Awgin serait, selon lui, l'indication d'un remaniement du texte et d'une addition postérieure. J.M. Fiey, Nisibe, p. 154. S. Brock, « Notes on some monasteries », p. 12.

25. Cité par J.M. Fiey, Nisibe, p. 155-156.

26. S. Brock, « Notes », p. 11-14.

27. J.M. Fiey pense qu'il s'agit d'une Vie très tardive sans « indication historique sérieuse », Nisibe, p. 156. 
Le couvent d'Awgin se trouve plusieurs fois cité par l'auteur du Livre de la chasteté, au VIII' siècle ; Isho 'denah raconte qu'Abraham de $\mathrm{M}^{\prime}$ arré en aurait réparé les ruines, à l'orée du VIII siècle (son successeur Ruzbihan fut métropolite de Nisibe en 726$)^{28}$. Cet élément permet de dire qu'autour de l'an 700, le couvent de Mar Awgin était syro-oriental. Et s'il fallait accorder quelque crédit à l'histoire de Mar Sallara, on pourrait dire que le couvent existait déjà au VII siècle ; il serait tombé en ruines dans le dernier quart de ce siècle - mais l'allusion à des agrandissements et à des constructions qu'aurait effectués Sallara semble en contradiction avec la désaffection totale qu'évoque Isho 'denah, à moins qu'une contingence extérieure tragique (raid, pillage, catastrophe naturelle, accident...) ne soit à l'origine de la démolition des bâtiments et du départ des moines. Cette indication chronologique serait corroborée par un court texte encore inédit signalé par S. Brock au Père Sabino Chialà : Dadisho' Qatraya, qui vécut à la fin du VII siècle, évoquerait des éléments de la légende eugénienne ; ainsi trouve-t-on cités la venue en Perse de l'ascète à l'époque du roi Shapur (II), le séjour dans une grotte ou la construction d'un monastère à Izla ${ }^{29}$. Mais en l'absence de tout élément de datation concernant ce texte, il paraît aventureux de le tenir pour probant.

À la faveur de la reconstruction des ruines de ce couvent, l'histoire d'un Mar Awgin père de moines se sera sans doute construite : les syro-orientaux auraient développé, vraisemblablement à partir de la figure d'un ascète ayant donné son nom à l'édifice et autour duquel se seraient groupés quelques disciples, une légende qui servit de support au rayonnement du «nouveau » couvent. L'affiliation d'Awgin au prestigieux monachisme égyptien éludait peut-être, comme l'a montré J. M. Fiey, une histoire monastique locale, autochtone, en Mésopotamie et plus largement en Perse, au bénéfice d'une parenté estimée plus glorieuse ${ }^{30}$.

28. J.-B. Chabot, Chasteté, p. 56 ; trad. p. 47, § 106. Voir S. Brock, « Notes », p. 1-6. Sur Ruzbihan, voir J. M. Fiey, Nisibe, p. 135.

29. Commentaire inédit sur le Paradis des Pères. S. Chialà, Abramo di Kashkar, p. 16 et n. 11.

30. Sur Awgin comme personnage historique, voir l'opinion de J.M. Fiey, « Aonès », p. 72-73, p. 81 ; id., Jalons, p. 104. 


\section{La tradition syro-orthodoxe}

Certains chercheurs ont estimé qu'il avait pu exister avant le VIII siècle une tradition syro-orthodoxe sur le personnage d'Awgin, tradition qui aurait été remaniée lors d'un réinvestissement du couvent par des syro-orientaux ${ }^{31}$. Néanmoins, la documentation ne permet pas de tenir pour assurés une présence syro-orthodoxe antérieurement à cette date et donc le façonnement d'une légende par les jacobites avant les syro-orientaux ${ }^{32}$. Les sources ne donnent en effet aucune indication quant à un changement d'occupation avant la refondation du couvent sur les ruines existantes autour de 700. J. M. Fiey avait émis l'hypothèse que le centre d'élaboration et de diffusion du culte d'Awgin et de sa légende chez les syro-orientaux aurait été le couvent de Mar Miha'il dans lequel Ruzbihan, successeur d'Abraham de M'arré (le restaurateur du monastère de Mar Awgin), se forma à la vie monastique ${ }^{33}$. Du côté jacobite, il propose, sans toutefois argumenter, que le village d'Enhel au Tur 'Abdin aurait pu être l'épicentre de cette composition car les habitants de la bourgade prétendaient avoir été convertis par le dernier disciple d'Awgin, Isha'ya, auteur d'une biographie de son maître ${ }^{34}$. Peut-être peut-on penser que l'un des développements de la légende qui attribue à Mar Malka, le neveu d'Awgin, une fondation à Enhel a contribué à la diffusion du cycle eugénien chez les jacobites ${ }^{35}$. Nous constatons ainsi que les attestations d'une histoire d'Awgin chez les syrooccidentaux sont postérieures à celles des milieux syro-orientaux.

31. J. Labourt, Le christianisme, p. 312-313. La présence syro-orientale est attestée jusqu' au XVII e siècle, J.M. Fiey, « Aonès », p. 77 n. 2.

32. Cf. aussi J.M. Fiey, Jalons, p. 103-104.

33. J.M. Fiey, «Aonès », p. 75-76.

34. J.M. Fiey, Jalons, p. 108. Cette information apparaît en note dans un abrégé du Livre de la Chasteté, mais elle reste conjecturale compte tenu des conditions de copie, J.M. Fiey, « Aonès », p. 77.

35. En fait, ni église ni couvent de Mar Malka ne sont signalés à Enhel dans les sources. Ce nom, qui signifie «la vallée» ou «le torrent» $n h l$, a pu être interprété a posteriori comme un site de fondation. Voir l'hypothèse de J.M. Fiey, Nisibe, p. 141, n. 48. Vie de Malka, P. Bedjan, Acta Martyrum V, p. 421-469. 
Les disciples d'Abraham de Kashkar et d'Awgin

Il existe une coïncidence entre les premières traces de la légende eugénienne et l'action de Mar Abraham de Kashkar : le mouvement réformateur inauguré par ce dernier au mont Izla, qui devait avoir une incidence capitale pour le développement du monachisme en Perse $^{36}$, survient historiquement au moment où commence à apparaître la figure de Mar Awgin dans le même secteur géographique. S. Chialà avait déjà pressenti que la revalorisation d'Awgin pouvait avoir quelques liens consécutifs à l'essor du mouvement initié par Abraham $^{37}$. Des éléments montrent des superpositions évidentes entre d'une part la tradition d'Abraham et de l'autre celle dite eugénienne, alors en plein essor, qui permettraient de proposer l'hypothèse d'une construction de la légende eugénienne à partir de l'histoire du fondateur du Grand monastère au mont Izla. Un regard sur les disciples respectifs de chacun peut être signifiant.

Depuis l'étude du Père Fiey sur les listes des disciples d'Awgin ${ }^{38}$, il faut considérer, en prenant appui sur des contradictions internes de la Chronique de Séert qui retransmet vraisemblablement deux traditions différentes, que les énumérations comportant vingt-huit noms ${ }^{39}$ seraient plus anciennes et donc à privilégier sur celles qui en présentent soixante-douze, à l'instar de la vie syriaque d'Awgin publiée par P. Bedjan ${ }^{40}$. La première notice du Livre de la chasteté dans sa version actuelle conserve une énumération de quatorze disciples dont les deux sœurs d'Awgin ${ }^{41}$; six autres noms sont par

36. F. Jullien, Le monachisme en Perse, 2008, sous presse.

37. S. Chialà, Abramo di Kashkar, p. 19. J.M. Fiey constatait que le culte d'Awgin avait obtenu « droit de cité dans les hauts lieux des disciples d'Abraham, et surtout au couvent de Rabban-Hormizd », « Aonès », p. 64-65.

38. J.M. Fiey, Jalons, p. 104-111.

39. A. Scher, Chronique de Séert I/2, p. 251 [139].

40. A. Scher, op. cit. I/1, p. 235 [25]. P. Bedjan, Acta Martyrum III, p. 472473. On sait que ce chiffre symbolique est construit sur modèle des disciples du Christ en Lc X, 1, 7 ; multiple de douze, il renvoie bien entendu aux tribus d'Israël mais aussi aux assistants de Moïse en Ex XV, 27. Sur les 70 ou 72 disciples du Christ, cf. B.M. Metzger, « Seventy or Seventy-two disciples ?», p. 67-76.

41. J.-B. Chabot, Chasteté, p. 2 ; trad. p. 4, § 1. 
la suite repérables dans le texte. On peut considérer qu'il s'agit là d'éléments parmi les plus anciens de la tradition eugénienne telle qu'elle circulait alors au IX' siècle, à l'époque d'Isho 'denah de Basra.

Examinons les patronymes de ces disciples d'Awgin dans les listes dont nous disposons (voir tableau page suivante).

Des similitudes patronymiques sont observables entre certains disciples d'Abraham et d'Awgin. Tout en portant sur un petit nombre, ces homonymies semblent toutefois significatives. On relève en particulier les noms de Daniel, Babaï, Yohannan, Guiwarguis, Siméon, Yahb, Shalita et Yonan. Ces deux derniers patronymes figurent dans les listes tenues pour anciennes, mentionnés par la Chronique de Séert et par le Livre de la chasteté. De plus, la présence de Yohannan de Nehel ou d'Ukama est également à relever car il s'agit là sans conteste de disciples directs d'Abraham de Kashkar ${ }^{42}$.

D'autre part, nous constatons dans cette documentation une corrélation étroite entre plusieurs disciples d'Abraham et des sites attachés aux noms de ceux d'Awgin. Ainsi Yona qui, près du village de Hutir (hwtyr) dans le pays de Qardu, bâtit un monastère sur les vestiges d'une fondation attribuée à un disciple (non nommé) d'Awgin ${ }^{43}$. Ukama aurait lui aussi édifié un couvent sur un site eugénien : la caverne où vécut l'anachorète Yohannan de Kamul ${ }^{44}$, présenté par l'auteur du Livre de la chasteté comme un élève d'Awgin ${ }^{45}$. De même, ayant quitté le Grand monastère, Isho ‘sabran choisit de s'établir au couvent de Zarnuqa, dont la fondation est traditionnellement attribuée à Mar Aha, disciple de Mar Awgin ${ }^{46}$. Concernant Ja'qub le reclus, Isho'denah de Basra l'inclut dans sa liste des disciples d'Abraham (notice 14) ; il devait être plus tard considéré comme un disciple d'Awgin ${ }^{47}$. Ces deux sources contribuèrent à l'essor de la légende en choisissant d'intégrer dans le nouveau cadre eugénien des personnages relevant de cycles bien

42. J.-B. Chabot, op. cit., p. 7-9; trad. p. 8-10, § 14.

43. J.-B. Chabot, op. cit., p. 21 ; trad. p. 19-20, § 33.

44. Cf. A. Scher, Chronique de Séert II/2, p. 582 [262]-583 [263].

45. J.-B. Chabot, Chasteté, p. 4 ; trad. p. 6, § 7.

46. Il apparaît dans les énumérations réduites, J.-B. Chabot, op. cit., p. 3-4, trad. p. $6, \S 6$.

47. Cf. J.M. Fiey, « Aonès », p. 71 n. 2. 


\begin{tabular}{|c|c|c|}
\hline $\begin{array}{l}\text { Vie syriaque d'Awgin } \\
\text { Acta Martyr. III, } \\
\text { p. } 472-473\end{array}$ & Livre de la chasteté & Chronique de Séert \\
\hline 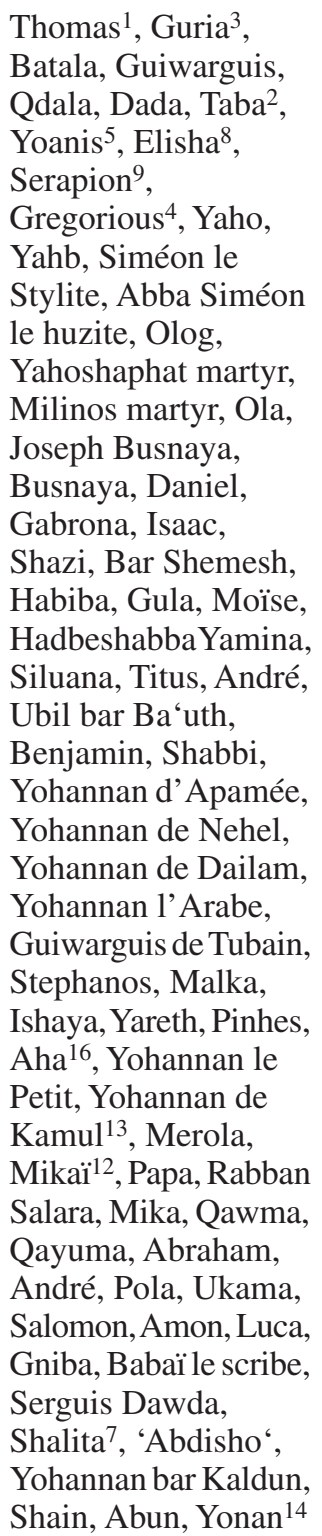 & $\begin{array}{l}\text { Thomas }^{1}(\S 1) \\
\text { Taba }^{2}(\S 1) \\
\text { Guria }^{3}(\S 1) \\
\text { Gregorious }^{4}(\S 1 ; \S 12) \\
\text { Yoanis }^{5}(\S 1) \\
\text { Yohannan }^{6}(\S 1) \\
\text { Shalita }^{7}(\S 1 ; \S 5) \\
\text { Elisha }^{8}(\S 1) \\
\text { Serapion }^{9}(\S 1) \\
\text { Yohannan }^{10}(\S 1) \\
\text { Sheri }^{11}(\S 1 ; \S 3) \\
\text { Mikail }^{12}(\S 1) \\
\text { Yohannan de Kamul }^{13} \\
(\S 7) \\
\text { Yonan l'anachorète } \\
(\S 4) \\
\text { Ezéchiel }^{15}(\S 84) \\
\text { Aha }{ }^{16}(\S 6) \\
\text { Yohannan du Beth- } \\
\text { Zabdaï }^{17}(\S 2) \\
\text { Safra } \\
\end{array}$ & $\begin{array}{l}\text { «Soixante-dix person- } \\
\text { nes le suivirent », I/1, } \\
\text { p. } 234 \text { [24]-[26] } \\
\text { « ... ceux qui vinrent } \\
\text { avec [Mar Awgin] au } \\
\text { désert d'Égypte étaient } \\
\text { au nombre de vingt- } \\
\text { huit », I/2, p. } 251 \text { [139] } \\
\text { Yonan }{ }^{14}, \mathrm{I} / 2, \text { p. } 246 \\
\text { [134]-250 [138.] } \\
\text { Yohannan de Beth- } \\
\text { Zabdé } 17, \text { I/2, p. } 250 \\
\text { [138]-251 [139] } \\
\text { Sari }{ }^{11}, \mathrm{I} / 2, \text { p. 251 [139] } \\
\text { Aha }{ }^{16}, \mathrm{I} / 2, \text { p. } 252 \text { [140] } \\
\text { Shalita }{ }^{7}, \mathrm{I} / 2, \text { p. } 259 \\
\text { [147] }\end{array}$ \\
\hline
\end{tabular}


précis, généralement liés à Abraham de Kashkar lui-même (sa descendance spirituelle, ou celle de moines artisans de sa réforme tels Bar 'Edta en Marga, Xvadahoy à Beth-Halé48, ou Ja'qub à Beth-'Abé). Ainsi en est-il de la liste des soixante-douze disciples du texte syriaque des Acta Martyrum (tableau col. 1), qui multiplie les anachronismes en vue de rattacher au cycle d'Awgin des figures spirituelles prestigieuses, ainsi maladroitement insérées parmi les proches de l'ascète.

À partir du vile siècle, la tradition d'Abraham se développe et se diffuse via les fondations par les moines partis du Grand monastère. Les sources qui retracent cette expansion du mouvement réformateur datent $\mathrm{du} \mathrm{IX}^{\mathrm{e}}$ siècle pour les plus anciennes. À cette époque, deux tendances se font jour : les auteurs qui ignorent encore la légende eugénienne - probablement parce qu'elle se développe au même moment - et ceux dont les écrits témoignent déjà d'une interpénétration avec cette tradition, comme le reflète la Chronique de Séert par exemple qui associe volontiers les deux figures lorsque sont présentées les fondations de monastères par les disciples d'Abraham. Les syro-orientaux vont contribuer à progressivement rattacher celui qui sera tardivement désigné comme « Père des moines », Awgin, à leur véritable « Père » historique, Abraham de Kashkar, à son tour inséré dans la lignée du plus récent désormais considéré comme l'initiateur de l'anachorèse en Mésopotamie. Les deux personnages cohabitent plus tard dans des hymnes liturgiques : ainsi une hymne sur Mar Awgin et ses disciples, mentionnée par 'Awdisho' de Nisibe et attribuée à Théodore de Merv, sans doute postérieure au XII siècle, chante-t-elle les louanges de cet anachorète, auxquelles sont associés des éloges rendus à Abraham de Kashkar ${ }^{49}$. Le manuscrit Add. 1979

48. Citons Ezéchiel, disciple de Xvadahoy connu par ailleurs par le Livre des supérieurs et qui vécut sous le patriarcat de Guiwarguis I ${ }^{\mathrm{er}}$ (661-680), E. Budge, The Book of Governors, I, p. 58-59 ; II, p. 102-103. Sur les problèmes liés à la recomposition de la notice d'Ezéchiel dans le Livre de la Chasteté, cf. J. M. Fiey, Assyrie chrétienne I, p. 23.

49. Cf. R. Duval, Littérature syriaque, p. 348 n. 1. Sa référence à G. Hoffmann est erronée. Cf. aussi J.S. Assemani, Bibliotheca III/1, p. 147, n. 4; W. Wright, Syriac Literature, p. 120, n. 4. S. Chialà signale également un passage de la Vie de Joseph Busnaya par Yohannan Bar Kaldun, du Xe-XIe siècle, où Awgin et Abraham sont célébrés ensemble comme lumières de l'Orient, Abramo di Kashkar, p. 18. 
de Cambridge comporte parmi les hymnes du mercredi une mention d'Abraham ${ }^{50}$; le moine apparaît au fol. 73a, après une citation d'Awgin et de son disciple Shalita (fol. 72ab). Sont évoqués dans l'ordre : Awgin, puis un peu plus loin Yohannan l'Arabe, Abraham de Kashkar, Babaï et Xvadahoy ${ }^{51}$.

Progressivement élaborée alors que se développe l'historiographie sur le personnage d'Abraham et son œuvre de réforme qui inaugurait un véritable renouveau pour le monachisme en Perse, la figure d'Awgin apparaît en partie comme un décalque du « Père des moines de l'Orient » qu'il finit par éclipser et même surpasser en notoriété, au point de devenir une référence commune pour les communautés chrétiennes.

Institut d'Histoire des Christianismes orientaux

Collège de France

52, rue du Cardinal Lemoine

F 75005 Paris

\section{BIBLIOGRAPHIE}

Joseph Simon AsSEMANI, Bibliotheca Orientalis clementino-vaticana IIII/2, Rome, 1719-1728.

Paul BeDJAN, Acta martyrum et sanctorum syriace I-VII, Paris, 1890-1897.

Sebastian BROCK, "Early Syrian monasticism”, Numen 20, 1973, p. 1-19.

Sebastian BROCK, "Notes on some monasteries on mount Izla", Abr-Nahrain 19, 1981, p. 1-19.

Ernest Walter Brooks, Eliae metropolitae Nisibeni Opus chronologicum

(Corpus Scriptorum Christianorum Orientalium 62*, script. syr. 21), Louvain, 1910 ; ibid. (Corpus Scriptorum Christianorum Orientalium 63*, script. syr. 23), Louvain, 1910.

50. W. Wright, Catalogue Cambridge I, p. 146, § 9.

51. Ce manuscrit fut rédigé en 2018 des Grecs (1707). Sur le culte d'Awgin dans les livres liturgiques syro-orientaux, J.M. Fiey, «Aonès», p. 64-65. Ainsi les deux cycles d'Awgin et de Rabban Hormizd sont-ils mêlés dans un évangéliaire de 1074 (ms. Add. 17923) qui ajoute à leurs côtés Abraham, son successeur à la tête du Grand monastère Dadisho', des fondateurs disciples d'Abraham $\left(\mathrm{n}^{\circ}\right.$ 148), Ja“qub de Beth-'Abé ( $\left.\mathrm{n}^{\circ} 152\right)$, Yozedeq ( $\left.{ }^{\circ} 142\right)$, etc. W. Wright, Catalogue British Museum I, p. 182-188, § CCXLVI. 
Ernest Alfred Wallis Budge, The Book of Governors. The Historia monastica of Thomas bishop of Marga A.D. 840 I-II, Londres, 1893.

Pierre CAnivet, Alice Leroy-Molinghen, Théodoret de Cyr. Histoire des moines de Syrie I-II (Sources Chrétiennes 234), Paris, 1977 ; ibid. (Sources Chrétiennes 257), Paris, 1979.

Jean-Baptiste СНАвот, Le livre de la chasteté composé par Jésusdenah, évêque de Baçrah (Mélanges d'archéologie et d'histoire 16), Rome, 1896.

Jean-Baptiste Снавот, «L'autodafé des livres syriaques du Malabar », Florilegium ou recueil de travaux d'érudition dédié à $M$. le marquis Melchior de Vogüé, Paris, 1909, p. 613-623.

Sabino Chialá, Abramo di Kashkar e la sua comunità, Magnano, 2005.

Rubens Duval, La littérature syriaque, Paris, 1907, réimpr. Amsterdam, 1970.

Jean Maurice FIEY, « Aonès, Awun et Awgin », Analecta Bollandiana 80, 1962, p. 52-81.

Jean Maurice FIEY, «Diptyques nestoriens du XIV siècle », Analecta Bollandiana 81, 1963, p. 371-409.

Jean Maurice FIEY, Assyrie chrétienne I-II, Beyrouth, 1965 ; III, Beyrouth, 1968.

Jean Maurice FIEY, "Ichô‘dnah, métropolite de Basra, et son œuvre », L'Orient syrien 11, 1966, p. 431-450.

Jean Maurice FIEY, Jalons pour une histoire de l'Église en Iraq (Corpus Scriptorum Christianorum Orientalium 31, Subsidia 36), Louvain, 1970.

Jean Maurice FIEY, Nisibe, métropole syriaque orientale et ses suffragants des origines à nos jours (Corpus Scriptorum Christianorum Orientalium 388, Subsidia 54), Louvain, 1977.

Jean Maurice FIEY, Dictionnaire d'Histoire et de Géographie Ecclésiastique 28, Paris, 2003, s.v. « Kashkar », col. 1021-1022.

Florence Jullien, Le monachisme en Perse. La réforme d'Abraham le Grand, Père des moines de l'Orient (Corpus Scriptorum Christianorum Orientalium, Subsidia), Louvain, 2008, sous presse.

Jérôme LABOURT, Le christianisme dans l'empire perse sous la dynastie sassanide (224-632), Paris, 1904.

Bruce MetzGer, « Seventy or Seventy-two disciples?», Historical and Literary Studies. Pagan, Jewish and Christian (New Testament Tools and Studies 8), Leiden, 1968, p. 67-76.

Alfonse Mingana, « Appendice [Histoire en vers du couvent de Sabrisho“ de Béth Koka] », Sources syriaques 1, Leipzig, 1907, p. 169-271.

François NAU, «Histoire d'Abraham de Kaskar et de Babaï de Nisibe », Revue de l'Orient Chrétien 21, 1918-1919, p. 161-172. 
Paul Peeters, « La légende de saint Jacques de Nisibe », Analecta Bollandiana 38, 1920, p. 285-373.

Addaï ScHER, Histoire nestorienne inédite (Chronique de Séert) I/1 (Patrologia Orientalis 4), Paris, 1908, p. 219 [9]-312 [102] ; ibid. I/2 (Patrologia Orientalis 5), Paris, 1910, p. 221 [109]-334 [222] ; ibid. II/1 (Patrologia Orientalis 7), Paris, 1911, p. 99 [7]-201 [109]; ibid. II/2 (Patrologia Orientalis 13), Paris, 1919, p. 437 [117]-636 [316].

Arthur VööBus, Syriac and Arabic Documents, regarding legislation relative to Syrian Asceticism (Papers of the Estonian Theological Society in Exile 11), Stockholm, 1960.

William Wright, Catalogue of the Syriac Manuscripts in the British Museum I-III, Londres, 1870-1872.

William WRIGHT, A short History of Syriac Literature, Londres, 1894.

William Wright, Catalogue of the Syriac Manuscripts in the Library of the University of Cambridge I-II, Cambridge, 1901. 